\title{
Raising the bar for clinical research
}

\author{
Nature Medicine is taking a comprehensive approach for reporting clinical studies with the aim to increase \\ transparency, promote rigorous reporting standards and engage with the community to meet the challenges of \\ contemporary medicine.
}

N ature Medicine has been at the forefront of publication of highquality translational research with the potential to change medicine for the past 25 years. Now, we aim to continue this commitment to leadership with the formalization of our guidelines for assessing and reporting clinical research. In doing so, not only do we reaffirm the journal's adherence to internationally accepted best practices and ethical standards, as invested stakeholders, we also aim to help to address the challenges of an evolving landscape of clinical practice that increasingly incorporates new scientific discoveries, technologies and big data.

Continuing our current practice and in alignment with guidance from the International Committee of Medical Journal Editors (ICMJE), all interventional clinical trials must be prospectively registered in a publicly available database that meets ICMJE standards. Prospective registration ensures transparency in clinical research. It discourages selective reporting of results, reduces research waste and, importantly, it provides patients, physicians and the public with access to information about ongoing clinical studies in which they might wish to participate. In the spirit of promoting transparency in reporting, registration of other study types, such as observational cohorts and meta-analyses, is encouraged but not required. To enable adequate evaluation of clinical studies by editors and referees, it is critical that authors also provide the approved study protocol with the manuscript at submission; and for trials registered at the start of 2019 and going forward, a data-sharing plan must be included in the trial registration as well as in the manuscript. All manuscripts published in Nature Medicine already include a Data Availability Statement. In the case of clinical studies the statement must summarize what data are available, as well as when and to whom, and how to access it, and clearly state any restrictions to data access.

The Enhancing the QUAlity and Transparency Of health Research (EQUATOR) network (http://www.equatornetwork.org) states that randomized clinical trials must be reported in accordance with guidance from Consolidated Standards of Reporting Trials (CONSORT). CONSORT guidelines were initially developed for randomized controlled trials, many of which are phase 2 and phase 3 studies. Although many early-phase and translational studies are not randomized, we believe that the basic CONSORT framework remains relevant in this context. As such, we encourage our authors to utilize the CONSORT guiding principles in the reporting of their non-randomized earlystage clinical trials. One example of a nonrandomized trial reported along CONSORT guidelines is a phase 1, first-in-human, dosing study of autologous macrophage therapy for liver cirrhosis from Forbes and colleagues, published in this issue. This study demonstrates safety and feasibility of a new cellular therapeutic strategy and lays the foundation for an ongoing phase 2 study with efficacy measures as the primary outcome. Other types of clinical research, including observational studies, biomarker studies and case reports, should be reported in accordance with the recommendations provided by EQUATOR for the respective study type.

As a journal at the interface between clinical and translational research we frequently consider reports of very earlystage clinical research, including discovery studies, and studies incorporating the power of new technologies in research and clinical practice. We are committed to developing best practices for reporting and publication of these new types of research as well. Discoveries in machine learning and artificial intelligence (AI), in particular, have matured beyond the laboratory and are being translated and implemented in clinical practice. Indeed, the US Food and Drug Administration has already started approving AI algorithms for interpretation of medical imaging. To address the challenge of reporting clinical research involving AI, in this issue the CONSORT-AI and SPIRIT AI groups announce the launch of an initiative to develop international, consensus-based, AI-specific extensions using the EQUATOR Network methodological framework. Contributions to the process from a wide range of stakeholders, including clinicians, trialists, computational experts, and others, such as funders and journal editors, are encouraged. A CONSORT-AI-specific extension is timely-and urgently needed-as the number of studies reporting the use of AI to address clinical challenges is growing exponentially, and the implementation of AI in medicine is only a matter of time.

Finally, our interests in best practices in clinical research are far-reaching beyond development and implementation of reporting standards. We are invested in engaging with the clinical community to meet new challenges in design, analysis and interpretation of clinical studies. One example is the debate about the value of real-world, observational data often derived from large datasets, such as the UK Biobank and electronic health records. The challenges of analyzing these data in a way that provides clinically robust and meaningful interpretations when compared with outcomes from randomized controlled studies are ongoing. In this issue, Dickerman and colleagues report statistical methodology to emulate randomized controlled trials from observational datasets. Their analyses can help to reduce confounding and biases inherent in these data types. It is important to acknowledge that this methodology is intended to be implemented in situations where it is unfeasible or unethical to perform a randomized controlled trial and is not intended to replace randomized trials, which remain the gold standard for testing clinical interventions.

We believe that our commitment to rigorous reporting standards for clinical studies is essential for maintaining the high quality of research published in the pages of Nature Medicine. Going forward, in partnership with our community, we will continue to refine these standards to ensure that they evolve as new technological and biological tools become available for translating scientific discovery to clinical impact.

Published online: 7 October 2019 https://doi.org/10.1038/s41591-019-0619-8 